\title{
Technology Evaluations on the TH-FACE Recognition System
}

\author{
Congcong Li, Guangda Su, Kai Meng, and Jun Zhou \\ The State Key Laboratory of Intelligent Technology and System, \\ Electronic Engineering Department, Tsinghua University, Beijing 100084, China \\ lcc@mails.tsinghua.edu.cn
}

\begin{abstract}
For biometric person authentication, evaluations on a biometric system are very essential parts of the entire process. This paper presents the technology evaluations on the TH-FACE recognition system. The main objectives of the evaluations are to 1) test the performance of the TH-FACE recognition system objectively; 2) provide a method to design and organize a database for evaluations; 3) identify the advantage and weakness for the THFACE recognition system. Particular description of the test database used in the evaluations is given in this paper. The database contains different subsets which are sorted by different poses, illuminations, ages, accessory, etc. Results and analysis on the entire performances of the TH-FACE recognition system would be also presented.
\end{abstract}

\section{Introduction}

Nowadays Biometric authentication has become one of the most active research areas in the world, as part of which, face recognition technology has made a large development. Therefore, How to evaluate the performance of the technology level among different systems or algorithms and how to test the adjustability to different conditions such as various poses, illuminations, ages, etc. have become urgent and difficult problems in front of researchers on this field. Moreover, evaluations can help customers to understand, to adopt and to identify a new technology [9].

There are already some famous evaluations on face recognition on the world, for example, the FERET [2] and the FRVT [1]. FRVT 2002 computed performance statistics on an extremely large data set; however, the images it used have not been distributed to the public. So FERET, which has publicly distributed its database containing fewer images than those in FRVT, has now been the standard testing set of international face recognition community.

Despite its success in the evaluations of face recognition algorithms, the FERET database has limitations in the relatively simple and unsystematically controlled variations of face images for research purposes [4]. Considering these limitations and aiming at the TH-FACE recognition system which primarily points to the oriental, we design the TH test database for the evaluations. The advantage of using such a database in the evaluations is that the results would more access the performance of the very system in real applications. Meanwhile, the technology evaluations on the 
TH-FACE recognition system cover different aspects on the system, including not only the recognition algorithms, but also the preprocessing methods.

The rest of the paper is organized as follows: Section 2 addresses the design of the evaluation including the contents of the TH test database and the subsets composing according to our design principles; Section 3 introduces briefly the TH-FACE recognition system. Section 4 presents the results and analysis of the evaluations on the TH-FACE recognition system. Finally, the conclusion is given in section 5 .

\section{Design of the Evaluation}

In this section, we would talk about the composition of the database used for evaluation and the design principles of the evaluations.

\subsection{Composition of the Database}

The TH test database contains 19,289 images of 750 individuals (394 males and 356 females) with controlled Pose, Expression, Glasses, Background and Lighting variations. All the images in the $\mathrm{TH}$ test database can be divided into two main subsets: the frontal subset and the pose subset.

- In the frontal subset, subjects in all the images are looking right into the camera that captures the images. Among the frontal images, each of the 750 subjects has a frontal image with normal expression, no accessory, standard lighting and plaint background. Every subject has another two images which were respectively captured one year and two year apart from the time when the normal frontal images mentioned above were captured. Some subjects may have images captured further before. There is also one image with complicated background outside the room. 554 subjects have images wearing glasses. All of the 750 subjects have images with expressions of a little smile.

- In the pose subset, every subject has $13(9+4)$ images with different poses. Among the 13 images of every subject, 9 images present that the subject is looking to the left, the central and the right, with a yaw angle from $-40^{\circ}$ to $+40^{\circ}$, each about $10^{\circ}$ apart, supposed the central is $0^{\circ}$ and the counter-clockwise is positive. The 4 remaining images and the frontal image of the 9 images mentioned just now, cover different pitching angles from $-20^{\circ}$ to $+20^{\circ}$, each about $10^{\circ}$ apart, supposed the central is $0^{\circ}$ and the counter-clockwise is positive.

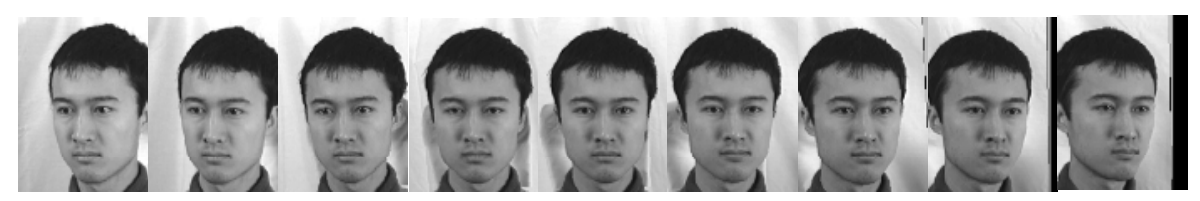

Fig. 1. Examples of left-right poses 
The content of the TH test database is summarized in Table 1.

Table 1. The contents of the TH test database

\begin{tabular}{c|l|c|c}
\hline \multicolumn{2}{c|}{ Subsets } & Subject \# & Image \# \\
\hline \multirow{4}{*}{ Frontal } & Normal & 750 & 750 \\
\cline { 2 - 4 } & Aging & 750 & 2,235 \\
\cline { 2 - 4 } & Glass & 554 & 554 \\
\cline { 2 - 4 } & Background & 750 & 750 \\
\cline { 2 - 4 } & Expression & 750 & 750 \\
\cline { 2 - 4 } & Lighting & 750 & 4,500 \\
\cline { 2 - 4 } & Yaw angles & 750 & 6,750 \\
\cline { 2 - 4 } & Pitching angles & 750 & 3,000 \\
\hline \multirow{2}{*}{ Pose } & Total: & 19,289 & \\
\hline
\end{tabular}

\subsection{Design Principles of the Evaluations}

A design principle describes how evaluations are designed. The precepts in these evaluations are:

1. The exploitation of the TH-FACE recognition system and the design of the evaluations are independently carried out;

2. Test data are not transparent at all to the TH-FACE recognition system before the evaluations.

3. Datasets used in the evaluations should reflect multiple performances of the system against different conditions.

Points 1 and 2 ensure the system is evaluated on their ability to generalize performance to new sets of faces, not the abilities of the system to be tuned to a particular set of faces [1]. Point 3 is up to the 'three bears' problem presented by Phillips, which sets guiding principles for designing an evaluation of the right level of difficulty. The goal in designing an evaluation is to have variation among the scores. There are two sorts of variation. One is variation among algorithms for each experiment, and the other type is variation among the experiments in an evaluation. Because at this time the evaluation was only taken on the TH-FACE recognition system, we emphasize on the latter.

According to these principles, we composed the two types of datasets below from the TH test database to implement the evaluations. All the images here can't be used for training in the system before. The datasets are summarized in Table 2.

- Gallery Set. A gallery set is a collection of images of known individuals against which testing images are matched. In the evaluation, the gallery set contains 750 images of 750 subjects (each subject has one image under normal condition). Actually, the gallery set consists of all the normal images mentioned in Table 1.

- Probe Set. A probe set is a collection of probe images of unknown individuals to be recognized. In the evaluation, 18 probe sets are composed from the TH-FACE 
database. Among them, 5 probe sets correspond to the 5 subsets in the frontal subset: aging, glass wearing, background, smile expression and lighting as described in Table 1. The other 13 probe sets correspond to the images with different poses.

Table 2. The datasets composed according the evaluation design principles

\begin{tabular}{|c|c|c|c|c|c|}
\hline \multicolumn{2}{|c|}{ Datasets } & \multicolumn{4}{|c|}{ Image \# } \\
\hline \multicolumn{2}{|c|}{ Gallery set } & \multicolumn{4}{|c|}{750} \\
\hline \multicolumn{2}{|c|}{ Datasets } & Image \# & \multicolumn{2}{|l|}{ Datasets } & Image \# \\
\hline \multirow{5}{*}{$\begin{array}{l}\text { Probe sets } \\
\text { (frontal) }\end{array}$} & Aging & 2,235 & \multirow{9}{*}{$\begin{array}{c}\text { Probe } \\
\text { sets } \\
\text { (different } \\
\text { yew } \\
\text { angles) }\end{array}$} & $-40^{\circ}$ & 750 \\
\hline & Glass & 554 & & $-30^{\circ}$ & 750 \\
\hline & Background & 750 & & $-20^{\circ}$ & 750 \\
\hline & Expression & 750 & & $-10^{\circ}$ & 750 \\
\hline & Lighting & 4,500 & & $0^{\circ}$ & 750 \\
\hline \multirow{4}{*}{$\begin{array}{l}\text { Probe sets } \\
\text { (different } \\
\text { pitching } \\
\text { angles) }\end{array}$} & $-20^{\circ}$ & 750 & & $+10^{\circ}$ & 750 \\
\hline & $-10^{\circ}$ & 750 & & $+20^{\circ}$ & 750 \\
\hline & $+10^{\circ}$ & 750 & & $+30^{\circ}$ & 750 \\
\hline & $+20^{\circ}$ & 750 & & $+40^{\circ}$ & 750 \\
\hline
\end{tabular}

\section{The TH-FACE Recognition System}

\subsection{MMP-PCA Face Recognition Method}

The baseline algorithm for the TH-FACE recognition system is called multimodal part face recognition method based on principal component analysis (MMP-PCA). Various facial parts are combined in this MMP-PCA method.

The algorithm firstly detaches face parts. According to the face structure, a human face is divided into five parts: bare face, eyebrow, eye, nose and mouth. Next principal component analysis (PCA) is performed on these facial parts to calculate the eigenvector of each facial part. The projection eigenvector of known human faces' facial parts are then stored in the database. In the face recognition procedure, the algorithm first calculates the projection eigenvalue of the human face, and then calculates its similitude degree with the projection eigenvalues stored in the database, after that sorts the faces in the database according to the similarity degrees from large to small. Display the photo and personal information of the person being searched according to this order.

By choosing all the facial parts or arbitrary several facial parts, the algorithm can be adjusted to gain a relative optimal recognition rate according to different situations.

\subsection{Preprocessing Method}

In the TH-FACE recognition system, the preprocessing of the face images includes several important steps: geometric normalization, illumination normalization and the process of removing the glasses. The details of these steps are described as follows: 
In the geometric normalization step, the TH-FACE rsystem automatically positions not only the eyes but also the chin point. Then each face image would be scaled and rotated so that the eyes are positioned in line and the distance between the chin point and the center of the eyes equals a predefined length. After that, the face image is cropped to a certain size which is $360 \times 480$ (pixels). Evaluation is taken to compare this method with the traditional method, which positions only eyes and makes the distance between the eyes equal to a predefined length.

In illumination normalization step, multi-linear algebra is applied to obtain a representation of face image, so separates the illumination factor from face images. Then illumination in different regions of the image can be compensated to a relative lighting balance so that decrease the bad effects caused by illumination. More details of the multi-linear algebra for illumination normalization can be referred to [11].

The TH-FACE recognition system uses a method to remove glasses on the face by combining the PCA reconstruction and compensating the face region hidden by the glasses with repeated iterativeness. The details of this method can be referred to [10]. The performance on using the glass-removal preprocessing method is shown in Fig. 2.

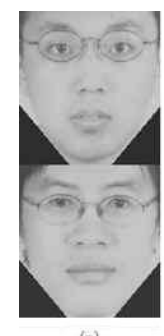

(a)

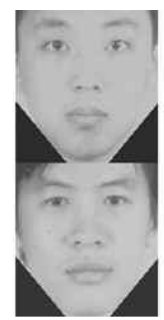

(b)

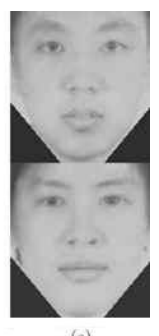

(c)

Fig. 2. Results of removing glasses in the TH-FACE recognition system

Images with glasses in the first line and those without glasses in the second line are both real images captured by camera. Image in the third line are synthesized from the first line of images by removing the glasses with the method mentioned above.

\section{Evaluation Results}

In this section, we carried out a set of experiments to evaluate the identification performances of the TH-FACE recognition system based on the datasets mentioned above. Effects caused by the preprocessing methods are also considered.

\subsection{Identification Rates from Different Probe Sets}

The face recognition system is evaluated on the 5 frontal probe sets and 13 pose probe sets as described in section 2.2. The statistic results of the identification rates in all these experiments are listed below. This performance statistics is described as follows: A probe has rank $k$ if the correct match is the $k^{\text {th }}$ largest similarity score. The identification rate at rank $k$ is the fraction of probes that have rank $k$ or higher [1]. 


\section{- Results on the frontal datasets}

From Fig.3 we can draw some conclusions: The TH-FACE recognition system generally has an excellent identification performance on frontal images with all the identification rates in the frontal sets above $70 \%$ for Rank 1 and above $80 \%$ for Rank 10. Especially, this system solves the glass wearing problem very well. In addition, the expression of a little smile does not impact the system too much, partly because of the MMP-PCA algorithm introduced in section 3.1, where mouse has the smallest projection eigenvalue. However, the system still needs to improve its adjustability to the changing of the lighting.

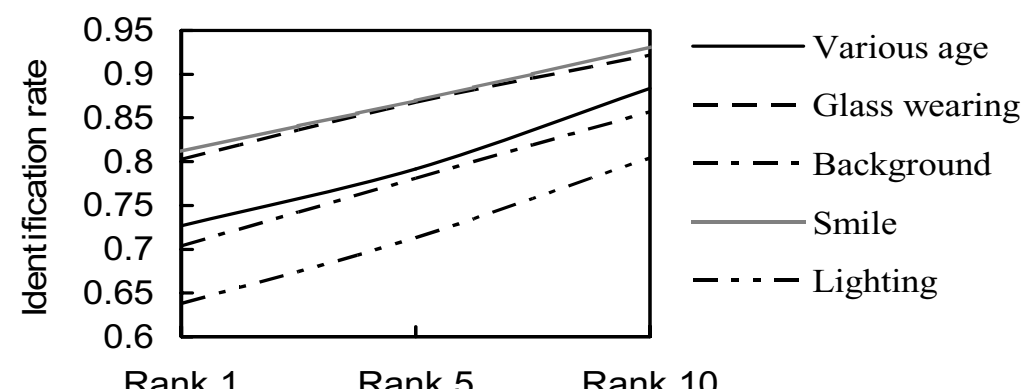

Fig. 3. The identification rates of the TH-FACE recognition system on different frontal datasets

\section{- Pose datasets}

Fig. 4 and Fig. 5 show the statistic results on different pose probe sets respectively. The angles in Fig. 4 are yaw angles which describe how left or right the subjects are looking to the camera while the pitching angle keeps $0^{\circ}$. In Fig. 5 the angles are pitching angles which describe how up or down the subjects are looking to the camera. The yaw angle keeps $0^{\circ}$ at that time.

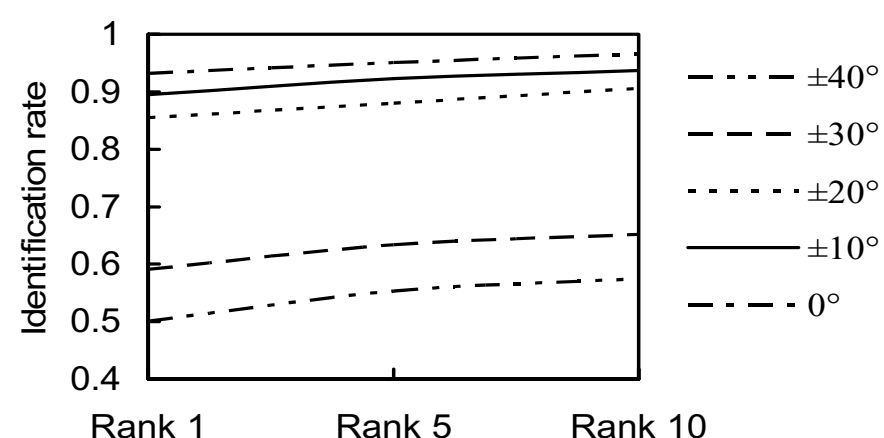

Fig. 4. The identification rates on different left-right pose probe sets 
From the results above, we can see that when the viewing perspective ranging from $-20^{\circ}$ to $+20^{\circ}$ (with reference to the vertical axis) the performance keeps relatively steady and high. While the angle increases more, the performance decreases rapidly.

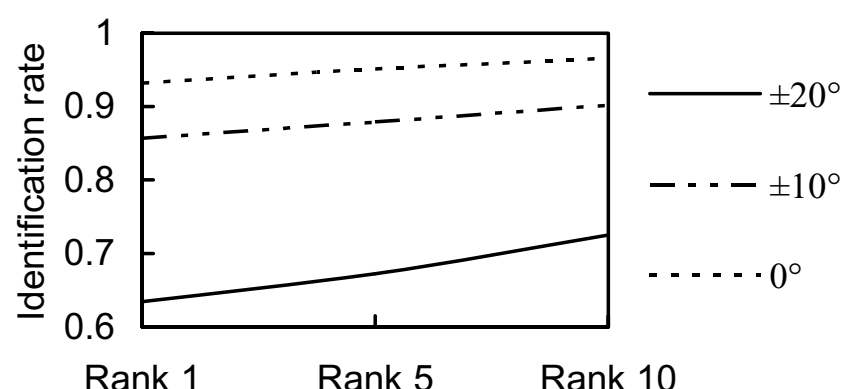

Fig. 5. The identification rates on different up-down pose probe sets

Similarly as the results on the left-right pose datasets, the results in Fig. 5 reflect that the identification works well while the pitching angle ranging from $-10^{\circ}$ to $+10^{\circ}$ while the performance decreases rapidly when the angle enlarges.

\subsection{The Performance Difference Caused by Preprocessing}

In this section, we examine the identification performance influenced by the preprocessing procedures.

In section 4.1, we can see that the TH-FACE system displays nice performance on the left-right pose datasets, which may be partly due to the geometric normalization method it chooses. So Evaluation is taken here to check the identification rate difference (choosing rate at Rank 1) under the two different geometric normalization methods mentioned in section 3.2. The result below shows the eyes-chin geometric normalization does positive effect on the identification rate indeed.

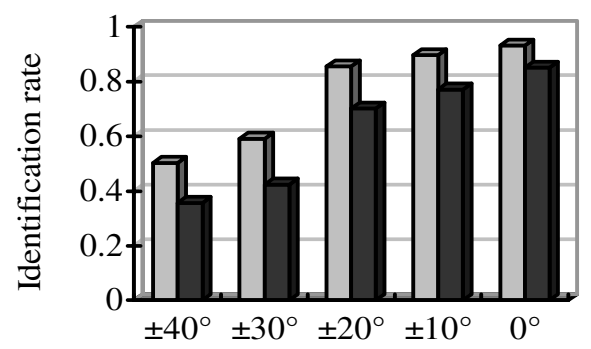

$\square$ eyes-chin geometric normalizatior

$\square$ eyes only geometric normalizatior

left-right pose datasets

Fig. 6. Identification performance according to different Geometric normalization 
We also carry out another evaluation on the effect of the glass removal preprocessing based on the glass wearing probe set. See results in Table 3. From the table above, we can easily understand the great positive effect of the glass removal preprocessing to the face identification performance while the system has to match an image with glasses among a gallery where all subjects are not wearing glasses.

Table 2. Identification performances with and without the glass removal preprocessing

\begin{tabular}{c|c|c}
\hline & $\begin{array}{c}\text { With glass removal } \\
\text { preprocessing }\end{array}$ & $\begin{array}{c}\text { Without glass removal } \\
\text { preprocessing }\end{array}$ \\
\hline Rank 1 & $80.3 \%$ & $37.3 \%$ \\
\hline Rank 5 & $86.8 \%$ & $41.8 \%$ \\
\hline Rank 10 & $92.2 \%$ & $45.2 \%$ \\
\hline
\end{tabular}

\section{Conclusion}

This paper presents the technology evaluations taken on the TH-FACE recognition system. The evaluations are based on the TH test database, containing 19,289 images of 750 individuals with controlled Pose, Expression, Glasses wearing, Background and Lighting variations. The division of database successfully achieves the object of finding out the performances of the system under different conditions. So this paper sets an example of technology evaluations on the TH test database and provides latest evaluation results on a new system with nice performance to the research community.

\section{References}

1. P. J. Phillips, P. Grother, R. J. Micheals, D. M. Blackburn, E. Tabassi, M. Bone: FRVT 2002 Evaluation Report, Technical Report.

Website: http://www.frvt.org/FRVT2002/documents.htm. March 2003.

2. P. J. Phillips, H. Wechsler, J. Huang, and P. Rauss: The FERET Database and Evaluation Procedure for Face Recognition Algorithms. Image and Vision Computing Journal, Vol. 16, No. 5 (1998) 295-306

3. Guangda Su, Cuiping Zhang, Rong Ding, Cheng Du: MMP-PCA face recognition method. Electronics Letters, Volume 38, Issue 25 (2002) 1654 -1656

4. Bo Gao, Shiguang Shan, Xiaohua Zhang, Wen Gao: Baseline Evaluations on the CASPEAL-R1 Face Database. Proceedings of the $5^{\text {th }}$ Chinese Conference on Biometric Recognition. (2004) 370-378

5. P. J. Phillips, H. Moon, P. Rauss, and S. Rizvi.: The FERET evaluation methodology for face-recognition algorithms. Proceedings Computer Vision and Pattern Recognition 97 (1997) $137-143$

6. Mansfield, T., G. Kelly, D. Chandler, and J. Kane.: Biometric Product Testing Final Report. Technical Report, Website:

http://www.cseg.gov.uk/technology/biometrics/index.htm. 2001.

7. D.M. Blackburn.: Evaluating Technology Properly - Three Easy Steps to Success. Corrections Today, Vol. 63 (1) (2001). 
8. Phillips, P. J., A. Martin, C. L. Wilson, and M. Przybocki.: An introduction to evaluating biometric systems. Computer, Vol. 33 (2000) 56-63.

9. P.J. Grother, R.J. Micheals and P. J. Phillips.: Face Recognition Vendor Test 2002 Performance Metrics. Proceedings of the 4th International Conference on Audio Visual Based Person Authentication, 2003

10. Cheng Du, Guangda Su.: Eyeglasses Removal from Facial Images. Pattern recognition letters. Accepted

11. Yuequan Luo, Guangda Su. A Fast Method of Lighting Estimate Using Multi-linear Algebra. Proceedings of $5^{\text {th }}$ Chinese Conference on Biometric Recognition (2004) 205-211 